\title{
Catheter-related right atrial thrombus and pulmonary embolism: A case report and systematic review of the literature
}

\author{
Karen EA Burns MD MSc FRCPC ${ }^{1,2}$, Andrew McLaren MD FRCPC ${ }^{3}$
}

KEA Burns, A McLaren. Catheter-related right atrial thrombus and pulmonary embolism: A case report and systematic review of the literature. Can Respir J 2009;16(5):163-165.

Central venous catheters (CVCs) are commonly used in clinical practice. One of the foremost complications associated with their use is the potential for symptomatic or asymptomatic thrombosis. CVC thrombosis, in turn, may not only result in vascular and catheter occlusion but also infection, pulmonary embolism, and formation of right heart thromboemboli. Thrombi within cardiac chambers are associated with an increased risk of mortality due to their potential for embolization to the pulmonary vasculature. We describe the case of a 77-year-old man, who was successfully thrombolyzed following detection of a right atrial thrombus and hemodynamically significant pulmonary embolism resulting from thrombus formation on the tip of a peripherally inserted central catheter (PICC). The present article is the first report of a PICC-related right atrial thrombus in an adult treated with thrombolysis. A systematic review of the literature suggests that the true incidence of this complication may be underestimated because the diagnosis may not be considered in asymptomatic and symptomatic patients, or may be missed by transthoracic echocardiography. The present case highlights the importance of maintaining a high index of suspicion for thromboembolic complications and heparin-induced thrombocytopenia in patients with CVCs or a PICC. It also underscores the important role of transesophageal echocardiography and thrombolysis in the diagnosis and management, respectively, of right heart thromboemboli with associated pulmonary embolism.

Key Words: Catheter; Echocardiography; Embolism; Heart; Thrombolysis

\section{CASE PRESENTATION}

A 77-year-old man with a medical history of chronic myelogenous leukemia, stable angina and chronic obstructive pulmonary disease was admitted for elective repair of a $5.8 \mathrm{~cm}$ juxtarenal abdominal aortic aneurysm, bilateral common iliac artery aneurysyms, and a right femoral thrombectomy. On the day of his surgery, his platelet count was $798 \times 10^{9} / \mathrm{L}$. Deep venous thrombosis (DVT) prophylaxis, with unfractionated heparin, was initiated within $24 \mathrm{~h}$ of surgery. The patient experienced hypotension that required fluid boluses on postoperative days 2 and 3. He began to mobilize on postoperative day 3. He continued to receive fluid boluses for low urine output and was noted to be confused and to have bibasilar crackles on auscultation. On postoperative day 9, he acknowledged shortness of breath and was noted to have leg edema. He appeared to have an elevated jugular venous pressure and a marginal blood pressure for which he received diuretics without effect. Subsequently, he developed fever, hypotension and worsening respiratory distress with bibasilar crackles and preserved oxygenation ( $91 \%$ on 2 L oxygen). After obtaining cultures, antibiotics for presumed sepsis were initiated. At this time, a

\section{Thrombus auriculaire droit et embolie pulmonaire liés à un cathéter : Rapport de cas et revue systématique de la littérature}

On utilise couramment les cathéters veineux centraux (CVC) dans la pratique clinique. L'une des plus importantes complications associées à leur emploi est le risque de thrombose symptomatique ou asymptomatique. La thrombose imputable à un CVC peut, en retour, entraîner non seulement une occlusion vasculaire et une occlusion du cathéter, mais également une infection, une embolie pulmonaire et la formation de thromboembolies au cœur droit. Les thrombi dans les cavités cardiaques sont associés à un risque accru de mortalité en raison du risque d'embolisation du réseau vasculaire pulmonaire. On décrit ici le cas d'un homme de 77 ans qui a subi avec succès un traitement thrombolytique, après quoi un thrombus auriculaire droit et une embolie pulmonaire hémodynamiquement significative ont été décelés suite à la formation d'un thrombus à l'extrémité d'un cathéter central inséré par voie périphérique (CCIP). Le présent article est le premier à faire état d'un thrombus auriculaire droit induit par un CCIP chez un adulte traité par thrombolyse. L'analyse systématique de la littérature donne à penser que l'incidence réelle de cette complication pourrait être sous-estimée car ce diagnostic n'est pas toujours envisagé chez les patients asymptomatiques et symptomatiques ou peut passer inaperçu à l'échocardiographie transthoracique. Ce cas illustre l'importance de maintenir un fort indice de suspicion à l'égard des complications thromboemboliques et de la thrombocytopénie induite par l'héparine chez les patients porteurs d'un CVC ou d'un CCIP. Il rappelle en outre le rôle majeur de l'échocardiographie transœsophagienne et de la thrombolyse dans le diagnostic et la prise en charge, respectivement, des thromboembolies du cœur droit associées à l'embolie pulmonaire. complete blood count revealed a hemoglobin of $66 \mathrm{~g} / \mathrm{L}$, a white blood cell count of $17.9 \times 10^{9} / \mathrm{L}$ and platelets at $284 \times 10^{9} / \mathrm{L}$.

On postoperative day 10 , the patient suffered a respiratory arrest that required intubation. Despite aggressive fluid resuscitation, he remained hypotensive, requiring hemodynamic support with noradrenaline and milrinone. Central venous vascular access was changed from a right-sided internal jugular central venous catheter (CVC) to a left-sided subclavian introducer, through which a pulmonary artery catheter was inserted. Initial hemodynamic parameters on milrinone demonstrated a central venous pressure of $10 \mathrm{mmHg}$, a pulmonary artery pressure of $42 / 26 \mathrm{mmHg}$, pulmonary capillary wedge pressure of $12 \mathrm{mmHg}$, a cardiac index of $4.2 \mathrm{~L} / \mathrm{m}^{2}$ and a systemic vascular resistance of $941 \mathrm{dyn} \bullet \mathrm{s} / \mathrm{cm}^{5}$. A complete blood count revealed a white blood cell count of $24.3 \times 10^{9} / \mathrm{L}$, hemoglobin of $82 \mathrm{~g} / \mathrm{L}$ and a platelet count of $124 \times 10^{9} / \mathrm{L}$. A contrast-enhanced computed tomography (CT) scan of his chest demonstrated multiple, bilateral pulmonary emboli (PE) (Figure 1). Compression ultrasonography failed to demonstrate the presence of DVT. Treatment for PE was initiated with unfractionated heparin. Results from blood cultures demonstrated methicillin-resistant Staphylococcus

${ }^{1}$ Interdepartmental Division of Critical Care Medicine, University of Toronto; ${ }^{2}$ Keenan Research Centre and Li Ka Shing Knowledge Institute,

St Michael's Hospital, Toronto; ${ }^{3}$ Division of Critical Care Medicine, London Health Sciences Centre - Victoria Hospital, London, Ontario

Correspondence: Dr Karen EA Burns, Critical Care Medicine, St Michael's Hospital, 30 Bond Street, 4-045 Queen Wing, Toronto, Ontario

M5B 1W8. Telephone 416-864-6060 ext 3567, fax 416-864-6013, e-mail burnsk@smh.toronto.on.ca 


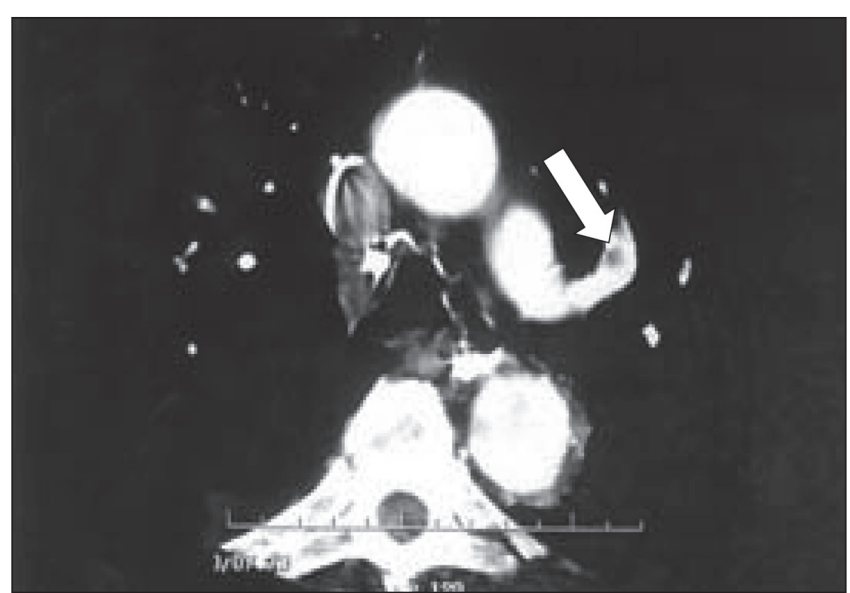

Figure 1) Pulmonary embolism. Contrast-enhanced computed tomography image demonstrating a filling defect (arrow) in the main left upper lobe artery

aureus and vancomycin was initiated. A left-sided internal jugular catheter and new arterial line were inserted. The patient's clinical status improved, and he was extubated on postoperative day 14 , at which time his platelet count was $87 \times 10^{9} / \mathrm{L}$. Oral coumadin was initiated the following day. A transthoracic echocardiogram (TTE) on postoperative day 15 showed a moderately to severely dilated right venticle (RV) with moderate RV dysfunction and mild tricuspid insufficiency. On postoperative day 17 , he was transferred to the ward and a new right subclavian CVC was inserted. The patient was noted to have increased swelling of his right arm two days later, and his international normalized ratio was 2.8. A 4 Fr double-lumen peripherally inserted central catheter (PICC) was inserted in the left cephalic vein on postoperative day 21 with its tip in the superior vena cava.

On postoperative day 22, the patient's oxygen requirements increased. The following day, his tachypnea worsened and he was noted to have a left lower lobe infiltrate on repeat chest radiography. At that time, his platelet count was $187 \times 10^{9} / \mathrm{L}$, with an international normalized ratio of 2.6 on coumadin and a prothrombin time of $48 \mathrm{~s}$. A repeat chest CT scan confirmed a new PE in the right upper lobe. Unfractionated heparin was resumed and an inferior vena cava filter was inserted. On postoperative day 24 , the patient was reintubated and vasopressor support was reinitiated. His platelet count was now $73 \times 10^{9} / \mathrm{L}$. A transesophageal echocardiogram (TEE) revealed a $2 \mathrm{~cm}$ serpiginous thrombus adherent to the tip of the PICC in the right atrium (Figure 2). The thrombus was rotating circumferentially within the right atrium (RA). A total of $100 \mathrm{mg}$ of recombinant tissue plasminogen activator was administered to the patient over $2 \mathrm{~h}$ through the PICC line initially (30 min), and subsequently through a peripheral intravenous line. A repeat TEE performed the following day showed resolution of the RA thrombus (RAT). The patient's platelet count was $85 \times 10^{9} / \mathrm{L}$ and a diagnosis of heparin-induced thrombocytopenia (HIT) was made by ELISA; fondoparinux was initiated. Oxygen and vasopressor support were discontinued over $48 \mathrm{~h}$ by postoperative day 27 and platelets had normalized the following day. The PICC line was retained and coumadin was reinitiated on postoperative day 41 . By postoperative day 43 , his platelet count had risen to $766 \times 10^{9} / \mathrm{L}$ and hydroxyurea was initiated.

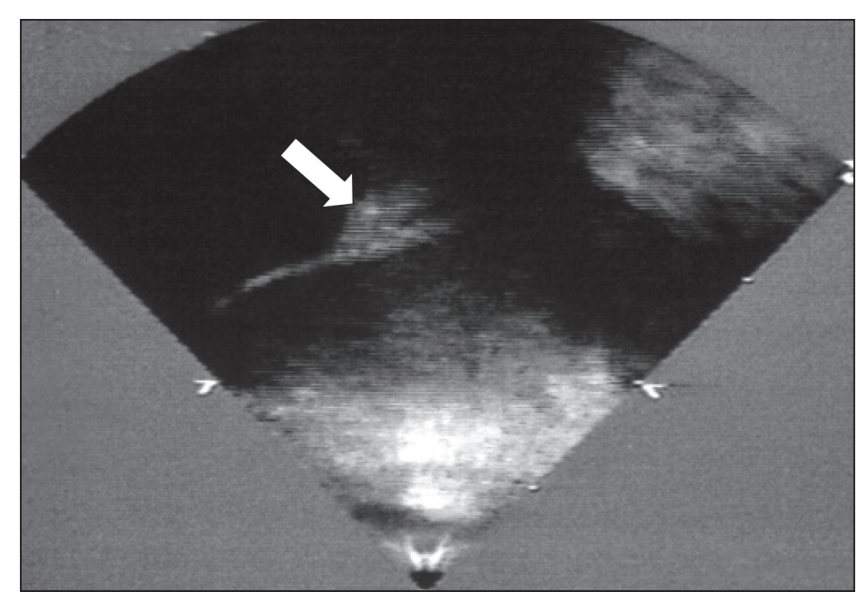

Figure 2) Right atrial thrombus attached to the central venous catheter. Transesophageal echocardiographic image demonstrating a clot (arrow) adherent to the distal end of a peripherally inserted central catheter in the right atrium

\section{DISCUSSION}

Thrombosis is a relatively common complication associated with CVC use. Inconsistent incident rates of CVC-related thrombosis are reported in the literature and vary according to host factors, catheter characteristics, cannulation site and the infusates administered. Estimates of CVC-related thrombosis vary depending on the site of insertion, with the incidence of PICC-related thrombosis, in general, ranging from $2 \%$ to $4 \%$. PE occurs in approximately $15 \%$ of individuals with CVCrelated upper extremity DVT.

Right heart thromboemboli (RHTE) are embolized DVTs lodged in the right heart chambers, predominantly the RA. RHTE also include thrombi that develop within the cardiac chambers at anastomotic foci or on injured endothelium, implanted devices or foreign bodies including tumours, pacemakers and CVCs. Classifications of RHTE are based on morphology. Type A thrombi are highly mobile and may prolapse through the tricuspid valve. Conversely, type B thrombi are attached to the right atrial or ventricular wall and may originate in association with foreign bodies or in structurally abnormal chambers. The incidence of right heart mural thrombi (innominate-superior vena cava, superior vena cava-RA and RA) was noted to be $29 \%$ in 141 patients with CVC undergoing postmortem examination (1). In a prospective study of 55 patients with CVCs who were followed for up to eight weeks, Gilon et al (2) noted the incidence of RHTE to be $12.5 \%$ and significantly associated with a catheter tip in the right atrium, malignancy, concurrent infection, procoagulant states and structural abnormalities. RHTEs are associated with PE in approximately $4 \%$ to $6 \%$ of cases $(3,4)$ and increase the three-month mortality rate from $16 \%$ to $29 \%$ (3).

While management strategies for hemodynamically significant PE have been well delineated, strategies for the management of RHTE, with and without PE, have not been well described. For patients with suspected PE, anticoagulation, in the absence of contraindications, should be initiated. Doppler ultrasonography of the lower and upper extremities, especially in the presence of a CVC, should be obtained. A confirmatory spiral CT or perfusion scan is desirable but may not be feasible in unstable patients. In hemodynamically unstable patients and patients with a CVC, an echocardiogram should be 
TABLE 1

Thrombolytic therapy for right atrial thrombi (RAT) associated with central venous catheters in adults

\begin{tabular}{|c|c|c|c|c|c|c|c|}
\hline $\begin{array}{l}\text { Author } \\
\text { (reference) }\end{array}$ & Clot location & Catheter & $\begin{array}{l}\text { Pulmonary } \\
\text { embolism }\end{array}$ & Treatment & Outcome & Clinical & Complications \\
\hline Imberti et al (7) & $\begin{array}{l}\text { SVC, extending } \\
\text { into right atrium }\end{array}$ & Right subclavian & No & $\begin{array}{l}\text { Catheter removal } \\
\text { rTPA }\end{array}$ & $\begin{array}{l}\text { RAT dissolved } \\
\text { SVC occluded }\end{array}$ & Improved & None \\
\hline Guindo et al (8) & SVC, right atrium & NA & No & $\begin{array}{l}\text { Catheter removal } \\
\text { Streptokinase }\end{array}$ & $\begin{array}{l}\text { RAT dissolved } \\
\text { SVC recanalized }\end{array}$ & NA & NA \\
\hline $\begin{array}{l}\text { Torbicki et al (3) } \\
\text { (ICOPER registry) }\end{array}$ & Right atrium & Yes & Yes & Streptokinase & NA & Short-term survival & $\begin{array}{l}\text { Gastrointestinal bleed } \\
\text { Transfusion }\end{array}$ \\
\hline Current case & Right atrium & PICC & Yes & rTPA & RAT dissolved & Improved & None \\
\hline
\end{tabular}

NA Not available; PICC Peripherally inserted central catheter; rTPA Recombinant tissue plasminogen activator; SVC Superior vena cava

obtained to assess for RV dilation and dysfunction, and RHTE. TEE is preferable to TTE because of its improved ability to detect RHTE and characterize clot morphology.

We considered three options in the management of our patient with RHTE, PE and hemodynamic compromise: surgery, interventional radiology-guided clot retrieval and thrombolysis. A surgical consult deemed that the patient was not a candidate for thoracotomy and cardiopulmonary bypass given his age, recent major surgery and hemodynamic instability. We subsequently approached interventional radiology to inquire about the possibility of clot and/or catheter removal; however, local expertise was not available. Consequently, we considered both systemic and catheter-directed thrombolysis for RHTE with PE as treatment strategies in our patient with RV dysfunction and hemodynamic instability. While several thrombolytic agents can be used to treat massive and submassive PE, we selected recombinant tissue plasminogen activator, which has a greater affinity for plasminogen in the presence of fibrin and a shorter infusion time than streptokinase or urokinase. We considered the risks associated with thrombolysis including bleeding, hematoma formation at puncture sites, intracranial hemorrhage, and the potential for proximal clot dissolution and subsequent embolization. While successful thrombolysis has been described for type A clots, type B clots may dislodge and cause PE following lysis of their attachments. Notwithstanding, the availability, low cost, ease of administration and comparable or superior survival associated with thrombolysis compared with surgery or heparin alone $(3,5,6)$ make it an appealing treatment option.

A registry (3) and systematic review (6) favour thrombolysis for the management of patients with RHTE. Notwithstanding, evidence supporting thrombolysis for the treatment of CVCrelated RAT, with or without PE, is limited to case reports. To summarize the current experience using thrombolytic agents to treat CVC-related RAT in adults, we systematically reviewed English language publications from Ovid MEDLINE (January 1966 to November 2004) using the following search terms: "exp catheterization/central venous" or "exp catherization peripheral, exp thrombosis”, "exp heart atria”, "exp vena cava”, "superior/ or exp vena cava", "inferior", 'right atri*.m[, exp fibrinolytic agents", "exp thrombolytic therapy/ or exp tissue plasminogen activator", "exp urinary plasminogen activator/ or exp streptokinase", "exp recombinant proteins". Additionally, we contacted the first author of a recent meta-analysis (3) of RHTE and PE to identify cases of CVC-related RAT treated with thrombolysis in their registry. Table 1 summarizes the case reports of thrombolytic treatment for RAT associated with CVCs in adults $(3,7,8)$. Limited information regarding the assessment of complications associated with thrombolysis was available. To our knowledge, the present article represents the first report of PICC-related RAT in an adult patient treated with thrombolysis.

The present case highlights several important considerations. First, CVCs expose patients to potential complications that, in turn, are associated with increased morbidity and mortality. Second, a decrease in platelets by $50 \%$ or more after day 4 and before day 14 should increase suspicion for HIT, even in the presence of concurrent sepsis. Third, several factors related to the host (eg, underlying chronic myelogenous leukemia), instrumentation (eg, PICC) and treatment (heparin exposure) may have acted synergistically and resulted in thrombus formation. Fourth, HIT in the presence of PE and a PICCrelated thrombus presents diagnostic and management challenges. Fifth, the literature may underestimate the incidence of thromboembolic complications related to CVC and PICC because the diagnosis may not be considered in symptomatic and, especially, in asymptomatic patients, or may be missed by TTE. Finally, the present case underscores the important role of TEE and thrombolysis in the diagnosis and management, respectively, of RHTE with associated PE.

ACKNOWLEDGEMENT: Dr Burns holds a Clinician Scientist Award from the Canadian Institutes of Health Research.

\section{REFERENCES}

1. Ducatman B, McMichan J, Edwards W. Catheter-induced lesions of the right side of the heart. A one-year prospective study of 141 autopsies. JAMA 1985;253:791-5.

2. Gilon D, Schechter D, Rein AJ, et al. Right atrial thrombi are related to indwelling central venous catheter position: Insights into time course and possible mechanism of formation. Am Heart J $1998 ; 135: 457-62$

3. Torbicki A, Galie N, Covezzoli A, Rossi E, De Rosa M, Goldhaber SZ; on behalf of the ICOPER Study Group. Right heart thrombi in pulmonary embolism: Results from the International Coooperative Pulmonary Embolism Registry. J Am Coll Cardiol 2003;41:2245-51.

4. Chakko S, Richards F III. Right-sided cardiac thrombi and pulmonary embolism. Am J Cardiol 1987;59:195-6.

5. Kinney EL, Wright RJ. Efficacy of treatment of patients with echocardiographically detected right sided heart thrombi: A metaanalysis. Am Heart J 1989;118:569-73.

6. Rose PS, Punjabi NM, Pearse DB. Treatment of right heart thromboemboli. Chest 2002;121:806-14.

7. Imberti R, Albertario F, Bellinzona G, Dionigi RV, Preseglio I, Mapelli A. Fibrinolytic (rt-PA) therapy for superior vena cava thrombosis in a multiple trauma patient. Acta Anaesth Belg 1991;42:233-6.

8. Guindo J, Montagud M, Carreras F, et al. Fibrinolytic therapy for superior vena cava and right atrial thrombosis: Diagnosis and follow-up with biplane transesophageal echocardiography. Am Heart J 1992;124:510-3. 


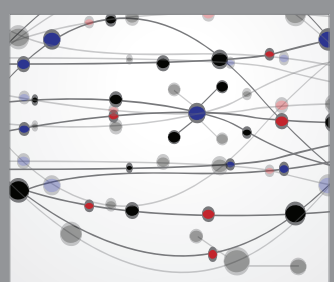

The Scientific World Journal
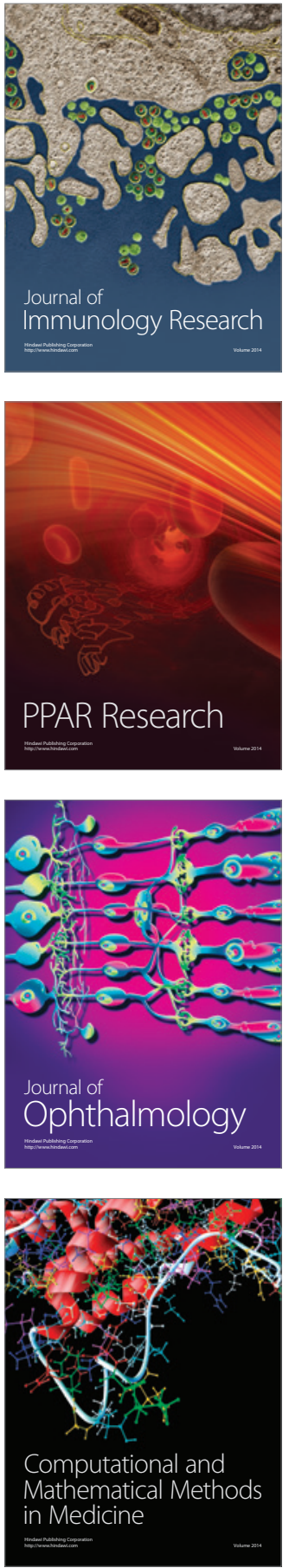

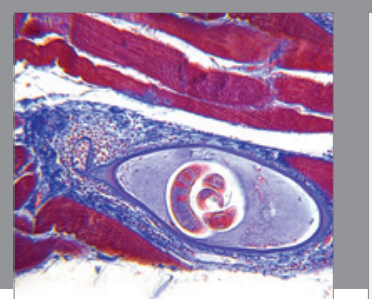

Gastroenterology Research and Practice

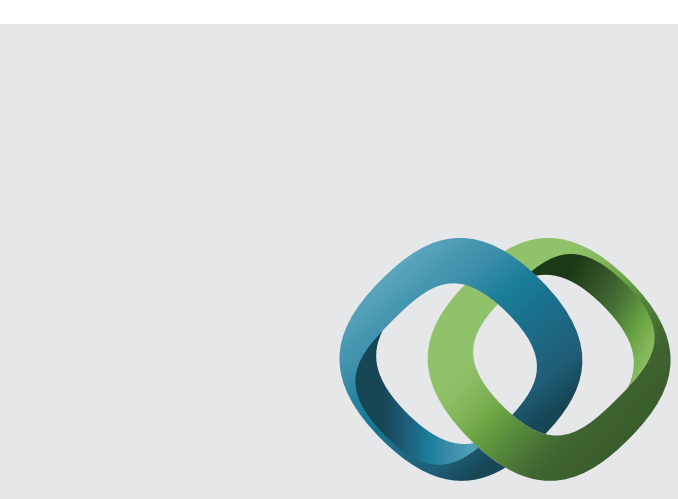

\section{Hindawi}

Submit your manuscripts at

http://www.hindawi.com
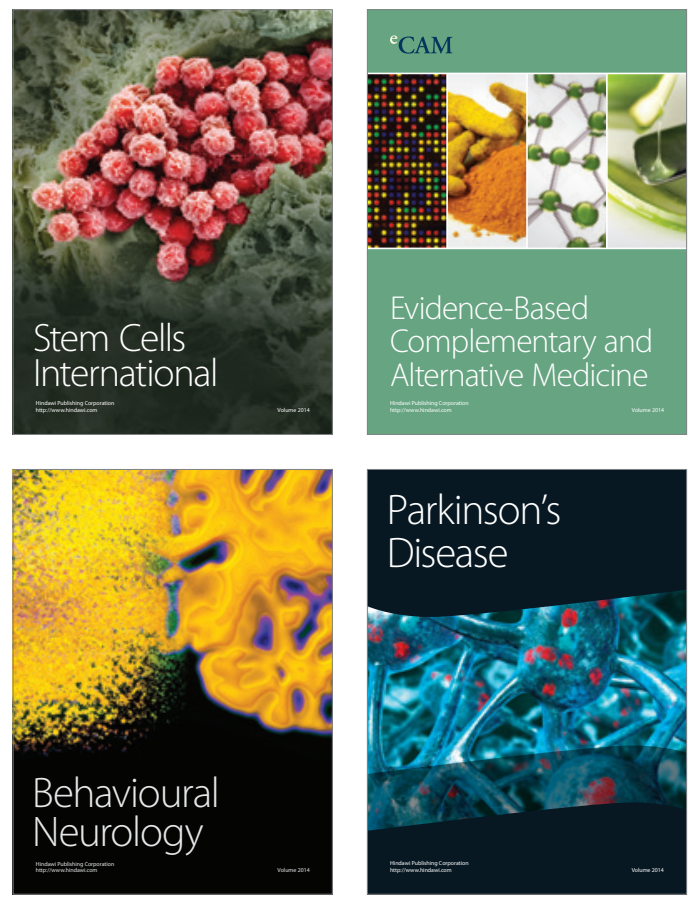
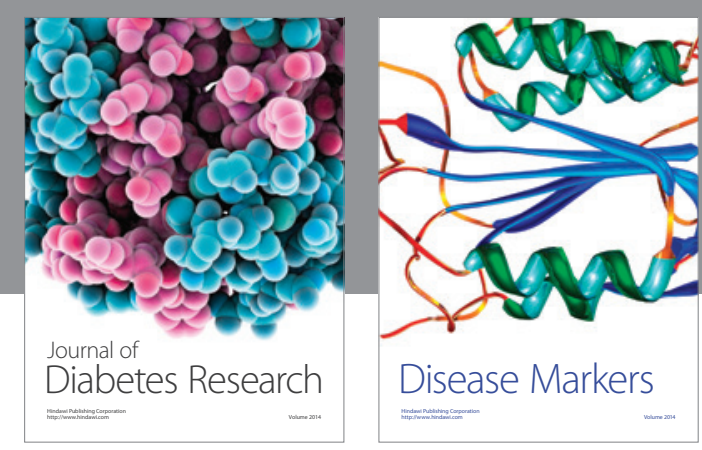

Disease Markers
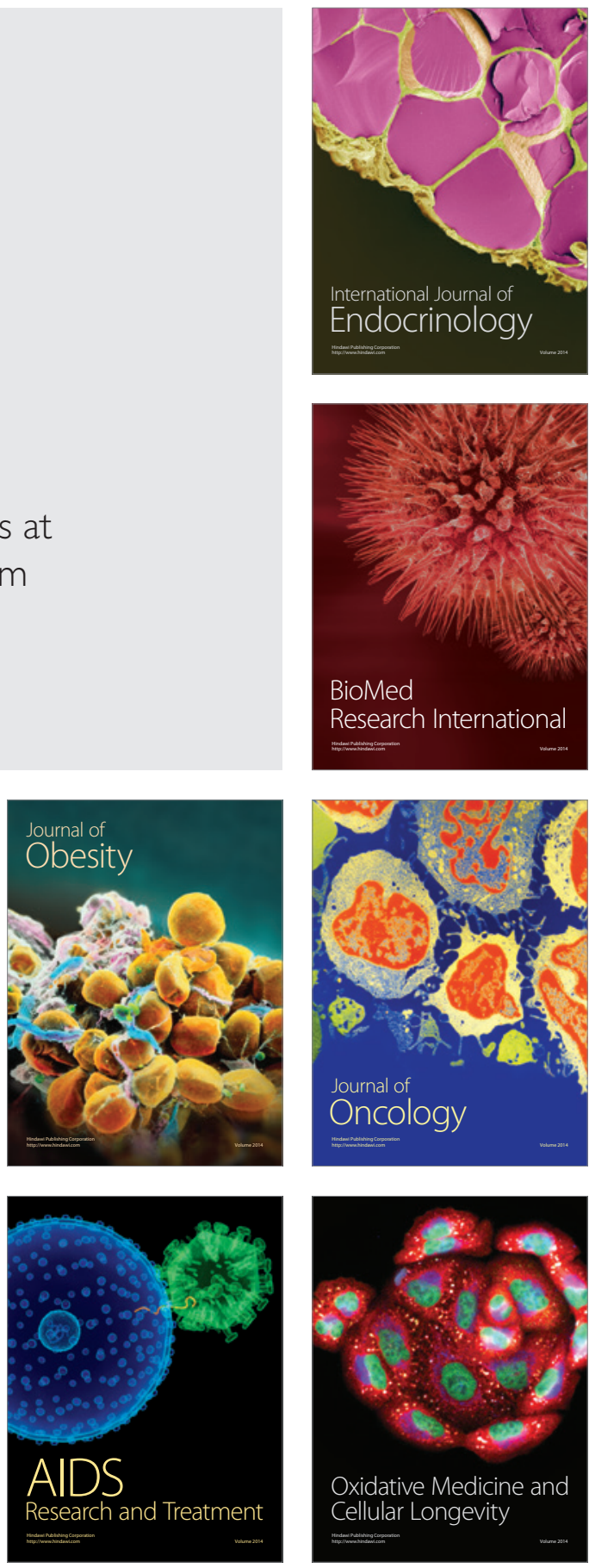